\title{
ERA-EDTA invests in transformation to greener health care
}

\author{
Peter J. Blankestijn ${ }^{1}$, Mustafa Arici ${ }^{2}$, Annette Bruchfeld ${ }^{3}$, Giovambattista Capasso ${ }^{4}$, Danilo Fliser ${ }^{5}$, \\ Denis Fouque ${ }^{6}$, Dimitrios Goumenos ${ }^{7}$, Markus Ketteler $^{8}$, Jolanta Malyszko ${ }^{9}$, Ziad Massy ${ }^{10}$, Ivan Rychlík ${ }^{11}$, \\ Goce Spasovski ${ }^{12}$ and Carmine Zoccali ${ }^{13}$ \\ ${ }^{1}$ Department of Nephrology, University Medical Center, Utrecht, The Netherlands, ${ }^{2}$ Department of Nephrology, Faculty of Medicine, Hacettepe \\ University, Ankara, Turkey, ${ }^{3}$ Department of Renal Medicine, Karolinska University Hospital, Stockholm, Sweden, ${ }^{4}$ Department of Nephrology, \\ University Campania 'Luigi Vanvitelli', Naples, Italy, ${ }^{5}$ Internal Medicine IV, Renal and Hypertensive Disease, University Medical Center, \\ Homburg/Saar, Germany, ${ }^{6}$ Department of Nephrology, Dialysis, Nutrition, Centre Hospitalier Lyon Sud, Pierre Bénite Cedex, France, \\ ${ }^{7}$ Department of Nephrology and Renal Transplantation, Patras University Hospital, Greece, ${ }^{8}$ Nephrologische Klinik Klinikum Coburg, Coburg, \\ Germany, ${ }^{9}$ Department of Nephrology, Dialysis therapy and Internal Medicine, Warsaw Medical University, Warsaw, Poland, ${ }^{10}$ Division of \\ Nephrology, Ambroise Paré Hospital, Paris Ile de France West University (UVSQ), Villejuif, France, ${ }^{11} 1$ st Department of Internal Medicine, \\ Third Faculty of Medicine, Charles University, Prague, Czech Republic, ${ }^{12}$ Department of Nephrology, Medical Faculty, University of Skopje, \\ Skopje, Former Yugoslav Republic of Macedonia and ${ }^{13} \mathrm{CNR}-\mathrm{IFC}$, c/o Ospedali Riuniti, Reggio Calabria, Italy
}

Correspondence and offprint requests to: Peter J. Blankestijn; E-mail: p.j.blankestijn@umcutrecht.nl, Twitter handle: @denisfouque1

\section{INTRODUCTION}

Living in an unpolluted environment is fundamental for human health and well-being. This general concept is obvious, but the interactions between environment and health/disease are very complex and truly arduous to assess scientifically. Air pollution, suboptimal water quality and poor sanitation are established threats to human health. However, it is increasingly clear that chemicals in the environment, noise, depletion of stratospheric ozone and degradation of soil constitute major problems for mankind. Respiratory, cardiovascular and kidney diseases, as well as cancer and reproductive and neurodevelopmental diseases, are all in part environmental disorders.

The relation between health care and environment/climate change is bidirectional. On the one hand, environment/climate change has identifiable effects on various aspects of human health, while the health care sector, when globally considered, also has a clear effect on the environment. It is estimated that between 5 and $10 \%$ of the global greenhouse gas emissions come from health care-related activities. This is in conflict with the general theme in medicine of 'primum non nocere'. While the attention of health professionals, policymakers and politicians to environmental problems in general is on the rise, the issue that the health care sector itself is a contributor to greenhouse gas emissions that affect the health of today's and future generations in a negative way is still largely ignored by most health professionals and by the community at large. One of the few exceptions is the UK, where the National Health Service put the subject on the agenda years ago. This document is a call for action to medical professionals, both as individuals and as representatives of professional organizations such as the European Renal Association-European Dialysis and Transplant Association (ERA-EDTA) to contribute to efforts aimed at establishing a more 'green' health care sector.

Several bodies have initiated activities on a global level, including the World Health Organization, the World Bank and others [1-3]. The Lancet Countdown is a collaboration between 24 academic institutions and intergovernmental organizations with representations from a wide range of disciplines [4]. In the title it calls for 'a global transformation for public health'. It tracks progress on health and climate change and provides an independent assessment of the health effects of climate change and the actions that are developed to stop it. It states: 'The health profession not only has the ability but the responsibility to act as public health advocates by communicating the threats and opportunities to the public and policy makers and ensuring climate change is understood as being central in human wellbeing'. Multiple levels of responsibilities can be identified (Table 1). Because health professionals are among the main stakeholders in health care, this community should accept its responsibility and contribute wherever and whenever possible by limiting the deleterious effects of greenhouse gas emissions generated in the health care sector. The national and multinational (European and global) organizations in which health care professionals are organized should have a clear and significant role in this. Health care professionals should ask themselves two crucial questions: 'How can my personal contribution to the health care system be done in a more environmentally friendly way?" and "How can my profession/professional organization contribute to make 
Table 1. Levels of responsibilities. Health care professionals need to take initiatives at all levels

- (Inter)national: global organizations, European Commission, governments, regulatory authorities, etc.

- Industries

- Hospitals and other health care providers: Institutions

Departments

Individuals:

As a civilian

As a professional:

'How can my personal contribution to the health care system be

done in a more environmentally friendly way?'

'How can my profession/professional organization contribute

to making health care more environmentally friendly?'

health care more environmentally friendly?' The central motive in addressing these questions should be 'lead by example'.

\section{HOW CAN ERA-EDTA CONTRIBUTE?}

The key message of the Lancet Countdown is the definition of 40 indicators (Panel 3 in the Lancet article). Organizations of health care professionals should recognize their responsibility and become, to the best of their ability, active in particular in areas represented by Indicators 3.9 and 5.2 (see section Concrete actions to be undertaken by the ERA-EDTA). The ERA-EDTA is the leading society in Europe for physicians working in the field of care of patients with kidney disease. The organization traditionally has a focus on a broad spectrum of activities aimed at improving the quality of patient care. Indicator 3.9 identifies the health care sector as an important contributor to greenhouse house emissions, whereas Indicator 5.2 identifies science as pivotal in increasing our understanding of the links between climate change and health. The Council of the ERA-EDTA recognized in its meeting in London on 22 February 2018, 'sustainability as a domain of quality in healthcare' and will therefore start to define actions to be undertaken by the organization to support the creation of carbon-smart health care. This means that the general goals defined by the global institutions should be translated into concrete actions and that the two above-mentioned indicators defined by the Lancet Countdown paper should be adopted as main focus areas for the ERA-EDTA, providing a positive contribution to this emerging problem.

Members of the ERA-EDTA traditionally are active in patient care, research and medical education. In all three sectors the subject of sustainability of health care should be on the table. Health care institutions should make up their own carbon footprint [i.e. the sum of greenhouse gas emissions released in relation to an organization, product or service, expressed as carbon dioxide equivalents $\left(\mathrm{CO}_{2} \mathrm{e}\right)$ ]. Awareness with in the medical profession of the various components that make up the carbon footprint is a start. This should be followed by initiatives on the institutional, departmental and individual levels to reduce the carbon footprint. Also research, both experimental and clinical, is environmentally unfriendly. Again, the first step is to increase awareness of the carbon footprint of the various activities. Further, funding and regulatory agencies should add a paragraph in their grant application forms/procedures on the environmental burden of a proposed research project and on possible strategies to reduce this burden.

ERA-EDTA members are often involved not only in the educational activities of medical students, junior doctors in training and nephrologists at early and late stages of their medical careers, but also of nurses and paramedical personnel. 'Sustainable health care education' is a teaching and learning method that prepares future health professionals to promote sustainable health and deliver sustainable health care. This should be part of the medical curriculum.

\section{CONCRETE ACTIONS TO BE UNDERTAKEN BY THE ERA-EDTA}

The main contributors to the carbon footprint of a hospital generally include (i) building: temperature regulation (heat and cold); (ii) electricity: light and medical equipment; (iii) transport: travel to and from the hospital of patients, visitors, employees, students, etc.; (iv) waste handling and (v) cleaning of textiles. Interventions aimed at to reducing the carbon footprint include general interventions that affect multiple departments or the whole institution and more specific interventions for certain specialities, such as nephrology. Some actions that should be undertaken by professionals and organizations such as the ERA-EDTA are summarized in Table 2.

Two examples within the nephrology field are haemodialysis and E-health. Haemodialysis is energy demanding, uses large quantities of water and creates substantial waste. Various dialysis providers have already undertaken initiatives in this respect. The ERA-EDTA will have an open mind in collaborating with industries to further strengthen these initiatives. A second example is: how to implement E-health, i.e. to develop and apply modern communication technologies on a much larger scale in nephrology, reducing the need for patients to come to the hospital. The EDA-EDTA could function as a platform for end users and information technology industries to support these initiatives. For these and other actions, we see multiple interactions with potentially relevant stakeholders (Figure 1).

\section{OPPORTUNITIES}

It is important to realize that accepting the responsibility to contribute to transformation to more sustainable health care also means that we can and should identify clear opportunities. In the short term, investments need to be made. However, it is very likely that in the long term initiatives will turn out to be cost reducing in this field. Indeed, large dialysis providers running multiple centres have already established remarkable savings by specifically addressing the subject. So far, in research, the effect of the environment on health and disease is a largely unexplored field. Exposome is the totality of external influences that humans experience every day. Modern data handling and management technologies make it possible to learn more on this. Also, the European Commission has opened calls to study the influence of environment on health and disease (Horizon 2020, Work Programme 2018-20, Part 8. Health, demographic change and well-being. Paragraph 1.5. Decoding the role of the 
(A) Actions to be undertaken by health professionals (adapted from [1])

Encourage the health facilities where you work to lead by example and adopt measures to reduce their climate footprint.

Encourage professional associations to explore and address the issue of climate change and the role the health sector can play in mitigation.

Work with associations of health professionals and teaching institutions to make climate literacy a mandatory requirement for all (pre)clinical education programmes.

Become an informed advocate for climate mitigation and adaption efforts.

Set an example: do your part to understand and minimize your own climate footprint.

\section{(B) Actions to be undertaken by professional organizations such as the ERA-EDTA}

Create awareness with the members of the enormous challenges that we are facing by inviting experts to give presentations during meetings or publishing topic papers in journals of the society.

Translate the general goals formulated by global institutions, such as the World Health Organization and World Bank into concrete actions within the specific medical fields.

Initiate, support and/or provide a platform for initiatives and/or task forces addressing specific topics, for discussions with other stakeholders, in particular industries, as well as policymakers, regulatory authorities, sister organizations, etc. on national and international level.

Initiate, support and/or provide a platform for initiatives and/or task forces for identifying areas within medical care that need attention most urgently, for instance, because these are especially heavy contributors to greenhouse gas emissions or because relatively simple actions could have an effect (low-hanging fruit concept). Prioritize areas to be addressed.

Initiate, support and/or provide a platform for initiatives and/or task forces for defining research questions on the effects of climate change on nephrology patients.

Initiate, support and/or provide a platform for initiatives and/or task forces for defining educational tools/programmes etc. for sustainable health care education.

Provide a platform for periodic reporting to the members, the general public and other stakeholders.

Organize meetings in a 'green' way.

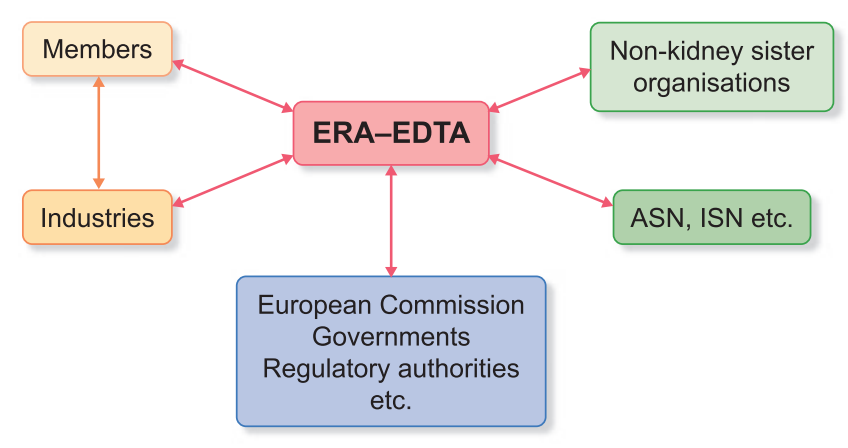

FIGURE 1: Interactions of ERA-EDTA with some stakeholders. ASN, American Society of Nephrology; ISN, International Society of Nephrology.

environment, including climate change, for health and wellbeing).

Finally, designing new educational tools and programmes making use of modern technologies should prepare health care workers for the future.

In summary, the ERA-EDTA as an organization, and also its members, should recognize and accept the responsibility, task and opportunity to 'translate' the general aims formulated by global institutions into concrete actions within the field of nephrology. Together, we need to become active contributors to the global task to transform to a more climate-smart health care sector and 'lead by example' (Table 2).

\section{CONFLICT OF INTEREST STATEMENT}

None declared. No conflict of interest related to this specific work, but individual conflict of interest forms can be viewed at http://era-edta.org/doi_.php.

\section{REFERENCES}

1. World Health Organization. Healthy Hospitals, Healthy Planet, Healthy People. http://www.who.int/globalchange/publications/healthcare_settings/ en/ (17 March 2018, date last accessed)

2. World Bank Group. Climate Smart Health Care. http://documents.world bank.org/curated/en/322251495434571418/Climate-smart-healthcare-lowcarbon-and-resilience-strategies-for-the-health-sector (17 March 2018, date last accessed).

3. Better Health. Better Environment. Sustainable Choices. Declaration of the Sixth Ministerial Conference on Environment and Health. Ostrawa, Czech Republic 13-15 June 2017; http://www.euro.who.int/_data/assets/pdf_file/ 0007/341944/OstravaDeclaration_SIGNED.pdf?ua=1 (17 March 2018, date last accessed).

4. Watts N, Amann M, Ayeb-Karlsson S et al. The Lancet Countdown on health and climate change: from 25 years of inaction to a global transformation for public health. Lancet 2018; 391: 581-630

Received: 19.3.2018; Editorial decision: 19.3.2018 